\title{
Short-Term Load Forecast for Port Harcourt Metropolis Using Artificial Neural Network.
}

\author{
Orie, Kenneth Eze ${ }^{1}$, Sigalo, Marvin Barivure ${ }^{2}$ \\ ${ }^{1,2}$ Center for Electrical Power System Research (CEPSR), Department of Electrical Engineering, Rivers State \\ University of Science and Technology,
}

\begin{abstract}
This paper presents a Short-term load forecast using Artificial Neural Network (ANN) method and applied it to Secretariat's 33KV feeder in Port Harcourt Metropolis electric power system. This predicts future load one hour in advance for 24 hours using past load readings data over the years, obtained from Port Harcourt Electricity Distribution Company (PHEDC) zonal office for the month of March and June 2015.The main stages include pre-processing of past data sets, network training and forecasting. The proposed Artificial neural network is composed of 3 layers: an input, a hidden, and an output layer consisting of six (6) and one (1) neuron in the input and output layers respectively, while that of the hidden layer vary for the different performance of the network, Levenberg-Marquardt (TRAINLM) training algorithm was used for the training process and a Mean squared error and Root mean squared error of $0.39 \%$ and $0.63 \%$ respectively were obtained when the trained neural network was tested on a week's data. This depicts a high degree of reliability of the proposed neural network to forecast load in PortHarcourt metropolis.
\end{abstract}

Keywords: Artificial neural network, Load Forecasting, Load demand, Multi-layer perceptron, Mean Squared Error.

\section{Introduction}

Port Harcourt Metropolis is a large and socio-economic city located in the South-South geo-political zone of Nigeria. Its inherent features depicts its feasible argument as the fastest growing city within the region in view of the oil exploitation and exploration activities in the area hence the astronomical rate of growth in its energy load demand (1).

Load forecasting is a technique used by power or energy providing companies to predict the power or energy needed to meet the demand and supply equilibrium. in essence; it is simply the prediction of future load/energy consumption or rate of energy consumption with the help of specific techniques or methods. In load forecasting accurate prediction of both magnitude and geographical location of electric load over wide range of planning in essentially taken into cognizance (2)Load forecasting can be grouped into three categories namely; short term, medium term and long term load forecast. Short term load covers duration of an hour to one week period; medium term covers duration of one week to one year, while long term covers duration above a year.STLF plays a key role in the formulation of economic, reliable and secure operating strategies for the power system (3)

Load forecasting helps an electric utility company to make important decision including decisions on purchasing generating electric power(4), load switching and infrastructure development. It plays a major role in the time control and security function of an energy management system. Accurate forecast greatly benefit power system planners to accomplishing a variety of tasks such as economics scheduling of generating capacity, scheduling of fuel purchases etc. in the deregulated economy, decisions on capital expenditures are also taken into consideration moree than in the deregulated economy where rate increases can be accounted for by capital expenditure projects (5) The importance of Accurate Load forecasting cannot be over emphasized due to the fact that it (load forecast) is an important component for power energy management system, paramount in unit commitment decision making, reduction in spinning reserve capacity, proper scheduling of various types of maintenance plan, and most importantly reduction in generation cost. Forecasted results are useful to system operators as a basis of off-line network analysis to determine if the system might be vulnerable. If so, correction action such as load shedding, purchase of power and introduction of peaking units on line, in time. The accuracy of forecasting is of great significance for the operational and managerial loading of a utility company. Evidently, a poor load forecast misleads planners and often results in wrong and expensive expansion plans. Equally, overestimating future electric loads may result in a redundant reserve of electric power. On the contrary, underestimation of load causes failure in providing sufficient electric power. For a planner to neither underestimate nor overestimate the load, convenient forecasting techniques with reasonable degree of accuracy need to be developed. The accuracy of load forecast on power system operation as it relates economy of operation and control of power system may result in errors during forecasting (6), (7), \& (8). These technique which can be numerical or AI algorithms such multiple linear regressions, time series, expert system, similar 
day approach, fuzzy logic, neural network, etc. The latter is been used in this research work due to its superior methodologies for short term load forecast compared to other traditional methods incorporating historical hourly load and temperature (9), the design of optimal network is not easily implemented and the fact that an electric load is a nonlinear function, traditional forecasting methods are not suitable for the application due to the lack of nonlinear mappingability .with optimal network structures and unified training algorithms, improved accuracy of forecast as well as performance of the network can be achieved via neural network approach.

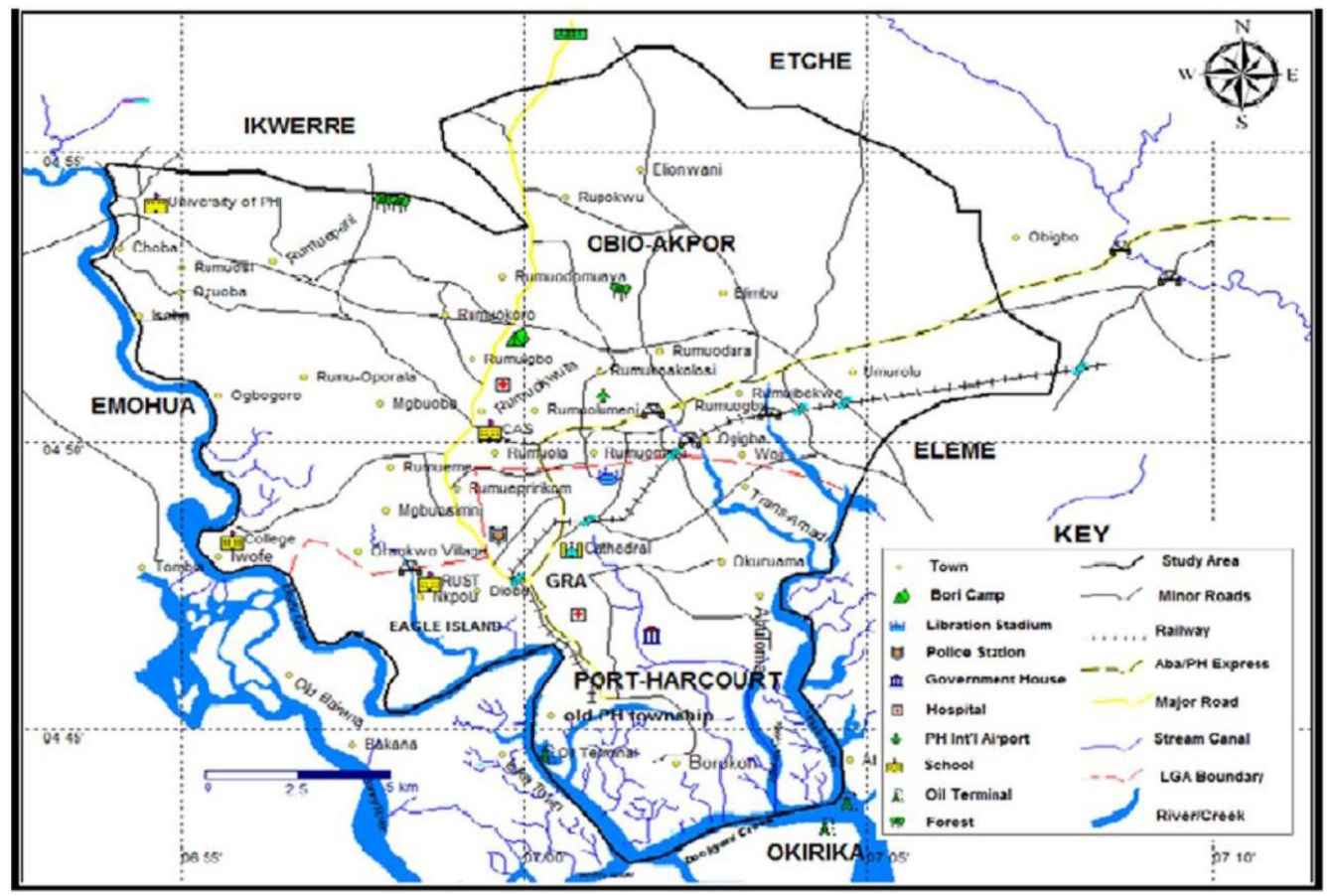

Figure 1.Geographical Map of Port Harcourt Metropolis, Rivers State, Nigeria.(10)

\subsection{Input Variables (Nomenclature)}

II. Variables

$\checkmark$ Time Hours of the day, Hour (Hr.)

$\checkmark$ PdlrshPrevious day hourly load reading in Mega-watts (MW)

$\checkmark$ PwhtempPrevious week hourly temperature in Degree-Celsius (0C)

$\checkmark$ PwlrmaxPrevious week maximum load reading in Mega-watts (MW)

$\checkmark$ PwlrminPrevious week minimum load reading in Mega-watts (MW)

$\checkmark$ PwlravPrevious week average load reading in Mega-watts (MW)

\subsection{Output Variable (Nomenclature)}

$\checkmark \quad$ Target $=$ Target $($ Expected output $)$ in Mega-watts (MW)

\section{Proposed Input-Output Schematic Diagram of System.}

Hour of the day
Preview day hourly
Load Reading
Previous day hourly temperature
Previous week maximum
Load reading
previous week minimum
Load reading
Previous week average
Load Reading

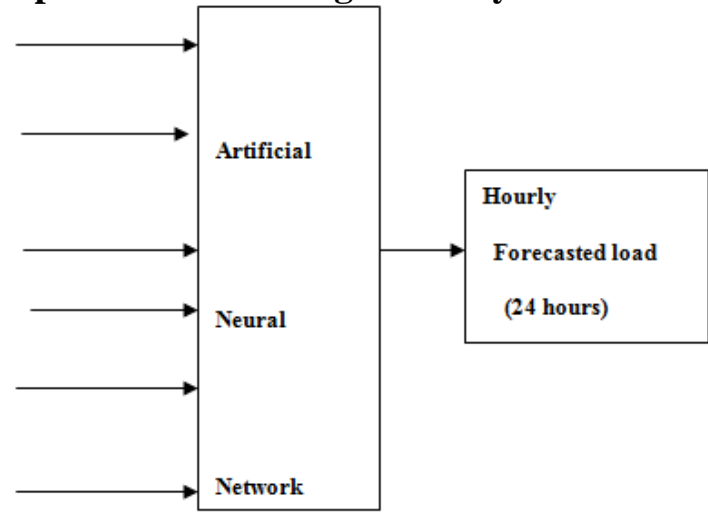

Figure 2. Input- Output Schematic Diagram of Proposed System 


\subsection{Multi-Layer Perceptron}

\section{Model}

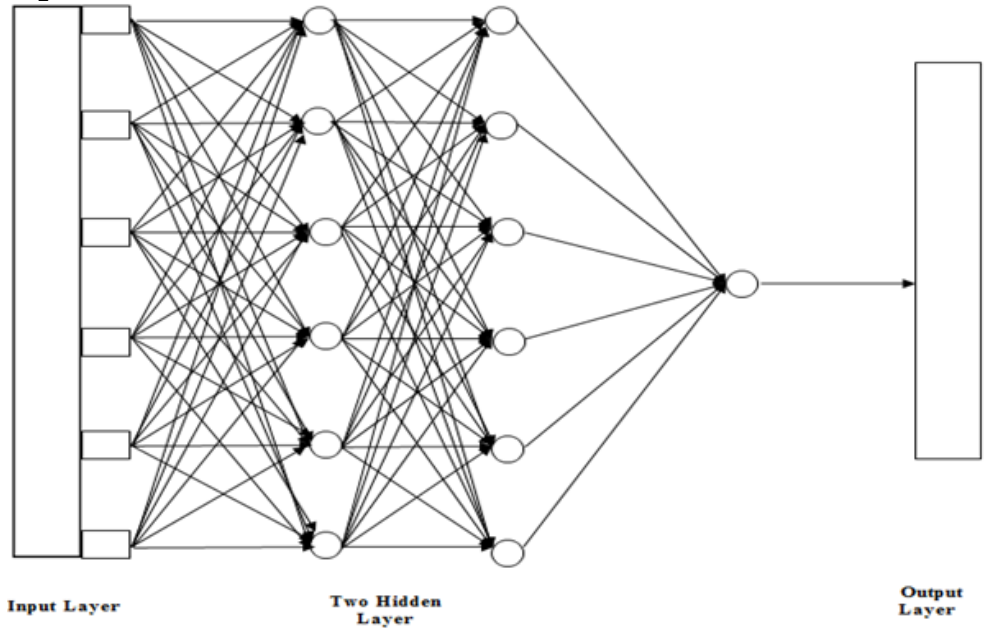

Figure 3: Block diagram of a two (2) hidden layer, six input multi-layer perceptron

\subsection{Summarized Training Parameters for Proposed ANN.}

$\checkmark$ Number of layers : three(3) (input, hidden, output layers)

$\checkmark$ Number of hidden layers: 1 to 2 (adjust as desired)

$\checkmark$ Number of neurons in hidden layer :10 to 60(adjust as desired)

$\checkmark$ Number of neurons in output layer : one (1)

$\checkmark$ Number of input parameters : six (6)

$\checkmark$ Activation function of hidden layer : logsig

$\checkmark$ Activation function of the output layer : linear

$\checkmark$ Training algorithm : levenberg-marquardt (trainlm)

$\checkmark$ Network parameter (train percentage): 70\% (16 sample)

$\checkmark$ Network parameter (validation percentage):15\% (4 sample)

$\checkmark \quad$ Network parameter (test percentage): $15 \%$ (4 sample)

$\checkmark$ Network performance : (Root\&Mean) Squared Error (RMSE\& MSE)

\section{Numerical Simulation Results and Discussion}

The proposed method has been implemented in MATLAB (R2013a) on a $2.10 \mathrm{GHz}$ core2Duo processor PC(10). Simulations were done using the proposed system and the trained Artificial Neural Network (ANN) was tested using the six (6) input variables for the month of June 2015, and an accurately hourly forecasted load for 24 hours was gotten according from table (3) comparison were made between forecasted load and actual load readings, graphs of forecasted verses actual load readings were plotted, as shown in figure (3) and finally percentage error (\%) were calculated, as shown in table (4)

Table 1. Input variables and target used in training proposed ANN.

\begin{tabular}{|l|l|l|l|l|l|l|}
\hline Time & Pdlrsh & Pwhtemp & Pwlrmax & Pwlrmin & Pwlrav & Target \\
\hline 1.00 & 9.4 & 21.0 & 9.7 & 6.0 & 8.3 & 8.4 \\
\hline 2.00 & 9.3 & 22.4 & 9.9 & 5.9 & 8.6 & 8.5 \\
\hline 3.00 & 9.2 & 23.0 & 10.0 & 5.8 & 8.4 & 8.4 \\
\hline 4.00 & 9.4 & 24.3 & 10.7 & 6 & 8.6 & 8.3 \\
\hline 5.00 & 9.6 & 25.8 & 10.8 & 6.1 & 9.0. & 9.3 \\
\hline 6.00 & 9.4 & 26.4 & 13.9 & 6.2 & 9.6 & 9.3 \\
\hline 7.00 & 9.2 & 27.6 & 12.0 & 9.2 & 10.9 & 10.0 \\
\hline 8.00 & 9.6 & 28.6 & 16.1 & 9.6 & 12.4 & 9.6 \\
\hline 9.00 & 10.0 & 29.0 & 17.8 & 9.2 & 13.5 & 9.5 \\
\hline 10.00 & 10.5 & 29.5 & 18.2 & 6.1 & 13.3 & 9.7 \\
\hline 11.00 & 10.8 & 30.0 & 19.0 & 6.0 & 13.9 & 9.9 \\
\hline 12.00 & 11.0 & 30.6 & 18.8 & 5.7 & 13.9 & 12.7 \\
\hline 13.00 & 12.0 & 30.0 & 18.6 & 6.2 & 13.8 & 13.6 \\
\hline 14.00 & 13.5 & 32.0 & 16.6 & 7.0 & 12.6 & 13.9 \\
\hline 15.00 & 13.4 & 31.5 & 18.6 & 6.7 & 12.1 & 14.0 \\
\hline 16.00 & 14.0 & 29.5 & 16.9 & 1.5 & 11.4 & 14.0 \\
\hline 17.00 & 12.0 & 28.0 & 15.8 & 2.8 & 10.1 & 13.9 \\
\hline 18.00 & 14.0 & 26.5 & 14.0 & 2.3 & 8.6 & 13.9 \\
\hline 19.00 & 17.4 & 25.2 & 17.4 & 2.5 & 10.6 & 15.5 \\
\hline
\end{tabular}


Short-Term Load Forecast for Port Harcourt Metropolis Using Artificial Neural Network.

\begin{tabular}{|l|l|l|l|l|l|l|}
\hline 20.00 & 17.6 & 24.4 & 17.6 & 6.7 & 12.3 & 15.6 \\
\hline 21.00 & 10.3 & 23.8 & 11.8 & 6.5 & 9.9 & 15.0 \\
\hline 22.00 & 10.0 & 23.8 & 11.1 & 7.2 & 9.7 & 14.0 \\
\hline 23.00 & 8.0 & 22.8 & 10.8 & 7.0 & 9.2 & 13.9 \\
\hline 24.00 & 5.5 & 22.5 & 10.7 & 5.5 & 8.6 & 12.6 \\
\hline
\end{tabular}

Table 1.Shows the hourly (24) values of the six input variables and target used in training the proposed artificial neural network for the $1^{\text {st }}$ day (Sunday)

Table 2. Inputvariables used for Testing Trained ANN

\begin{tabular}{|l|l|l|l|l|l|}
\hline Time & Pdlrsh & Pdhtemp & Pwlrmax & Pwlrmin & Pwlrav \\
\hline 1.00 & 11.5 & 21.0 & 14.1 & 0.9 & 10.4 \\
\hline 2.00 & 11.5 & 22.0 & 13.5 & 1.0 & 9.8 \\
\hline 3.00 & 12.0 & 22.0 & 13.3 & 1.0 & 9.8 \\
\hline 4.00 & 12.6 & 23.0 & 13.5 & 1.4 & 10.2 \\
\hline 5.00 & 12.9 & 24.0 & 15.7 & 1.5 & 11.2 \\
\hline 6.00 & 13.7 & 24.0 & 16.5 & 1.5 & 12.7 \\
\hline 7.00 & 15.1 & 25.0 & 17.6 & 1.3 & 14.0 \\
\hline 8.00 & 15.9 & 24.0 & 17.6 & 12.4 & 15.4 \\
\hline 9.00 & 15.2 & 24.0 & 19.5 & 12.9 & 16.6 \\
\hline 10.00 & 15.1 & 24.0 & 19.4 & 11.5 & 16.0 \\
\hline 11.00 & 15.3 & 24.0 & 19.6 & 11.1 & 16.0 \\
\hline 12.00 & 15.7 & 24.0 & 19.5 & 11.3 & 15.7 \\
\hline 13.00 & 11.1 & 24.0 & 19.4 & 11.1 & 15.5 \\
\hline 14.00 & 13.9 & 24.0 & 19.7 & 11.0 & 16.0 \\
\hline 15.00 & 13.3 & 27.0 & 19.7 & 11.2 & 16.0 \\
\hline 16.00 & 14.0 & 25.0 & 18.2 & 12.0 & 15.1 \\
\hline 17.00 & 13.3 & 26.0 & 16.9 & 11.2 & 13.4 \\
\hline 18.00 & 15.0 & 25.0 & 18.0 & 11.0 & 14.7 \\
\hline 19.00 & 14.9 & 25.0 & 17.5 & 1.3 & 12.2 \\
\hline 20.00 & 13.2 & 25.0 & 14.7 & 4.0 & 12.0 \\
\hline 21.00 & 13.7 & 24.0 & 19.8 & 5.3 & 13.1 \\
\hline 22.00 & 11.6 & 24.0 & 18.6 & 11.6 & 13.5 \\
\hline 23.00 & 11.4 & 24.0 & 17.0 & 11.4 & 14.0 \\
\hline 24.00 & 10.9 & 23.0 & 15.0 & 10.9 & 13.2 \\
\hline & & & & &
\end{tabular}

Table 2 shows the hourly values (24) of the six input variables used for testing the trained artificial neural network for the $1^{\text {st }}$ day (Sunday)

Table 3.24 hour Values of actual \& forecast load using trained Artificial Neural Network (ANN)

\begin{tabular}{|l|l|l|}
\hline Time & Actual & Forecast \\
\hline 1.00 & 7.2 & 7.7 \\
\hline 2.00 & 7.8 & 8.1 \\
\hline 3.00 & 7.5 & 7.9 \\
\hline 4.00 & 5.1 & 5.5 \\
\hline 5.00 & 5.4 & 5.9 \\
\hline 6.00 & 9.5 & 9.6 \\
\hline 7.00 & 7.5 & 7.7 \\
\hline 8.00 & 9.0 & 9.2 \\
\hline 9.00 & 12.8 & 13.0 \\
\hline 10.00 & 12.7 & 13.1 \\
\hline 11.00 & 13.8 & 13.8 \\
\hline 12.00 & 12.6 & 13.0 \\
\hline 13.00 & 6.7 & 6.9 \\
\hline 14.00 & 12.8 & 13.0 \\
\hline 15.00 & 7.9 & 8.2 \\
\hline 16.00 & 7.7 & 7.8 \\
\hline 17.00 & 4.0 & 4.4 \\
\hline 18.00 & 8.2 & 8.7 \\
\hline 19.00 & 12.2 & 14.3 \\
\hline 20.00 & 8.8 & 9.8 \\
\hline 21.00 & 7.7 & 8.4 \\
\hline 22.00 & 5.6 & 6.1 \\
\hline 23.00 & 4.2 & 4.4 \\
\hline 24.00 & 0.9 & 1.1 \\
\hline & & \\
\hline & & \\
\hline
\end{tabular}


Table 4. Percentage error values of trained Artificial Neural Network for seven (7) days.

\begin{tabular}{|l|l|l|}
\hline Day & MSE (\%) & RMSE (\%) \\
\hline Sunday & 0.34 & 0.58 \\
\hline Monday & 0.61 & 0.78 \\
\hline Tuesday & 0.99 & 0.10 \\
\hline Wednesday & 0.18 & 0.42 \\
\hline Thursday & 0.19 & 0.44 \\
\hline Friday & 0.36 & 0.60 \\
\hline Saturday & 0.11 & 0.33 \\
\hline
\end{tabular}

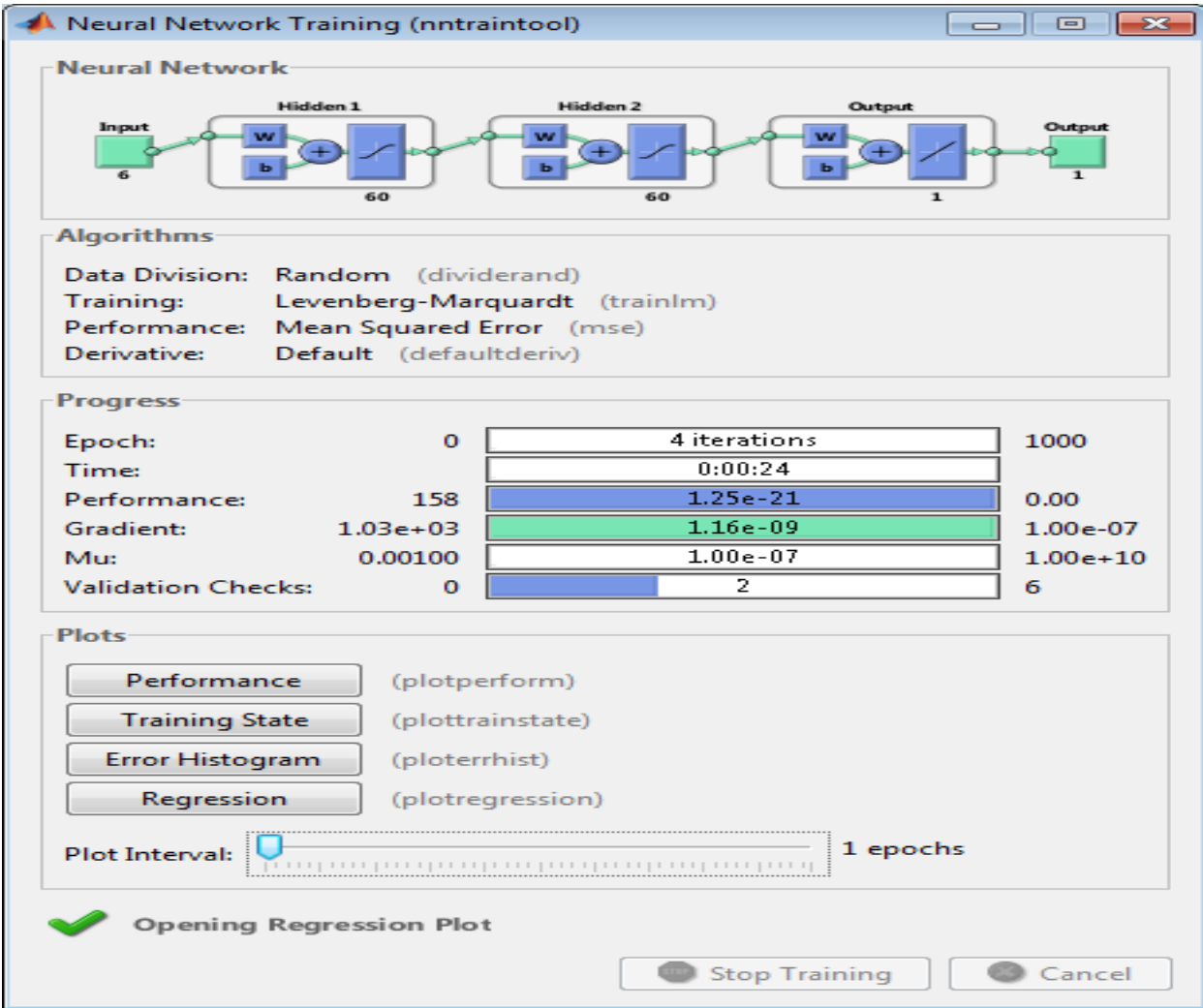

Figure 4. Neural Network Diagram Algorithm, Progress during Training

Figure 4.Shows the Neural Network Training interface using the neural network train tool.The interface display amongst other information, the neural network diagram, the training algorithm, the training progress and various plots as shown above.

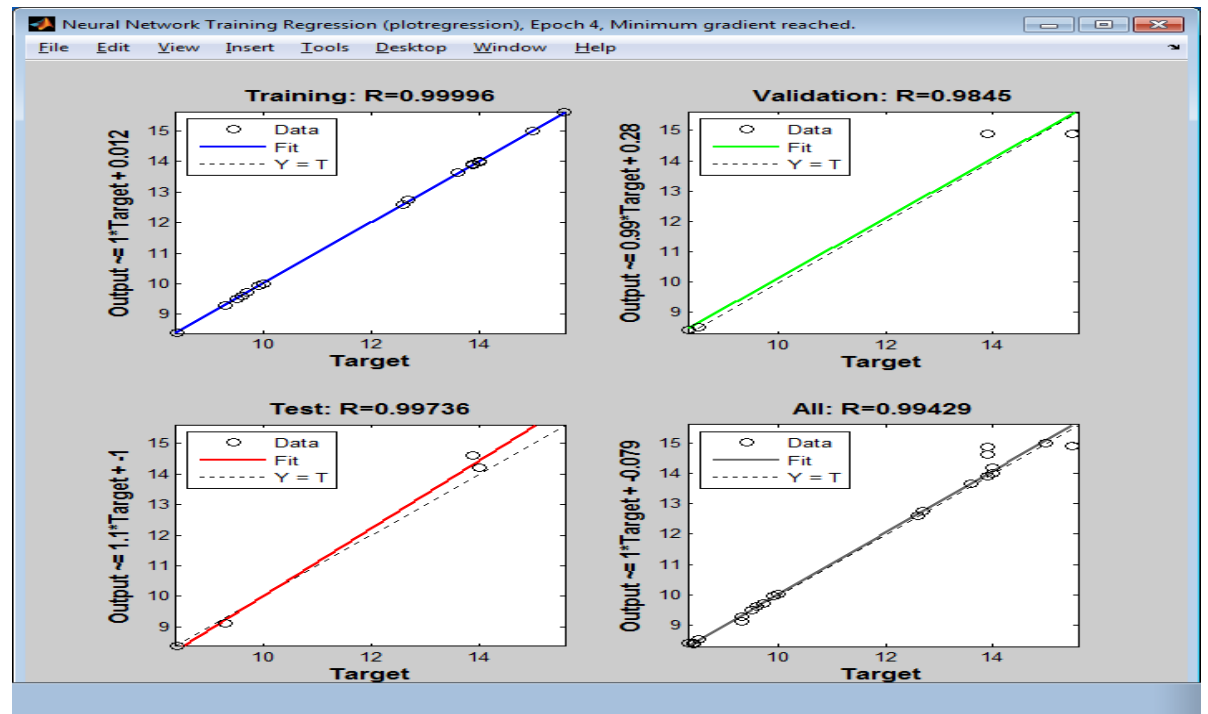

Figure 5: Regression Plot from Trained Neural Network. 


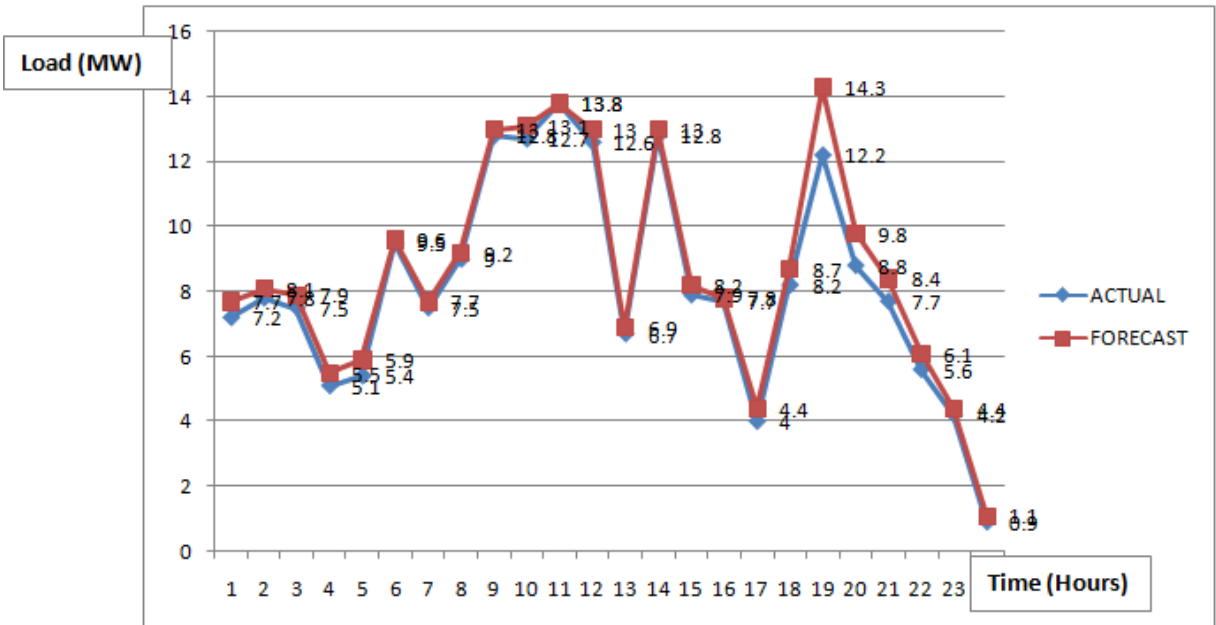

Figure 6: Graph of Actual Verses Forecast load after Testing Phase.

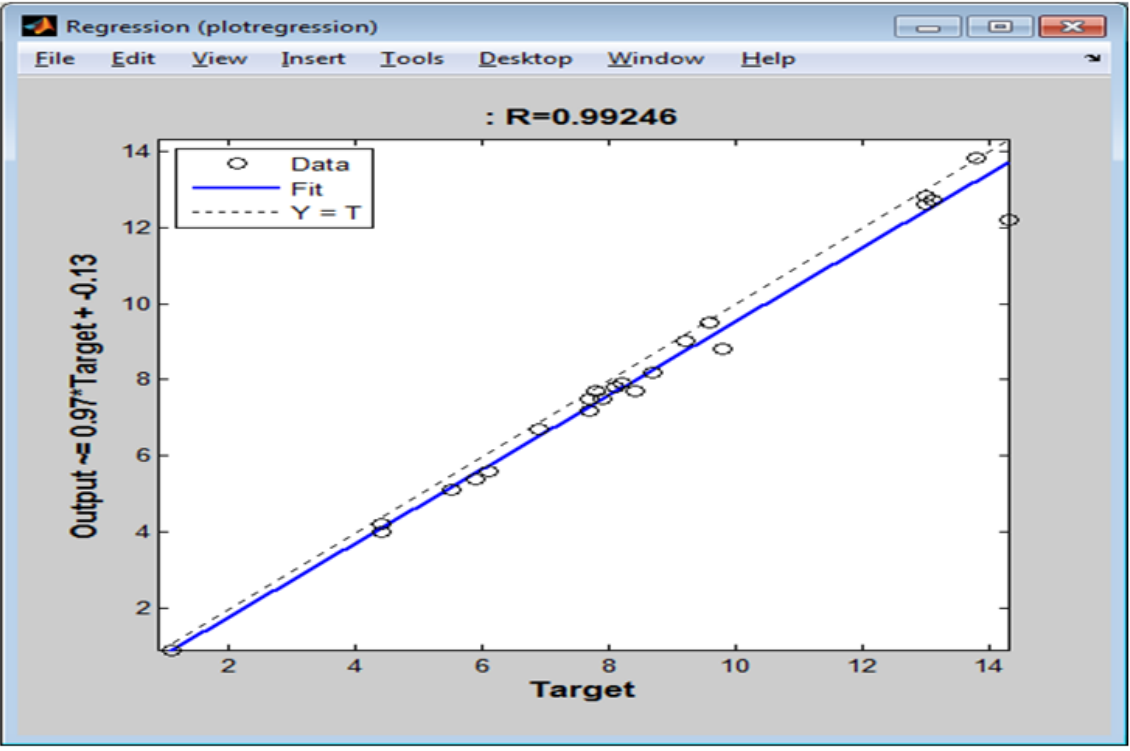

Figure 7. Regression plot of actual verses forecast load after testing phase using table 3 .

\section{Conclusion}

The results obtained in this research work from the regression plotand percentage error calculated for the seven days confirm the reliability as well as the efficiency of neural networks in short-term load forecast. The trainedneural networkhaving tested using a week's data was able to expose the non-linear relationship that existed among the hour of the day, previous day hourly load reading, previous day hourly temperature, previous week maximum load reading, previous week minimum load reading and previous week average load reading data supplied to it, at the training phase and with that make accurate prediction on what the load would be in secretariat $33 \mathrm{KV}$ Feeder in the next 24 hours, hourly.

\section{References}

[1]. Idoniboyeobu D. C., \& Newman M.A., (2014) "Electric load prediction technique using multiple regression method ; case study of Port Harcourt metropolis" European journal of scientific Research, vol. 118, no1, pp. 61-74

[2]. Rafal W. (2006) "modeling and forecasting electricity loads: a Statistical Approach". John Willey \& Sons Ltd.

[3]. (3) George, G., \& Francisco, D. (1987) "short- term forecasting” IEEE Transaction on power system, vol. 75, no. 12, December pp. $1558-15734$

[4]. Schlabbach, J. \& Kari-Heinz, R. (2008) “Power System Engineering”.Wiley \&Verley Co.

[5]. Kothari D.P., \&Nagrath, I.J. (2003) "Modeling and forecasting electricity loads" Tata McGraw Hill.

[6]. Eugene, A.F. \& Dora, G. (2005) "applied mathematics forPower systems: optimization, control and computational intelligence" Supinger science and business media, New York.

[7]. Rhman S., \&Hazim, O. (1993) "A generalized knowledge based short-term Load forecasting technique" IEE transaction on power system, vol .8, May,pp. 508-514.

[8]. Soliman, S.A., \& Ahmad, M.A. (2010) "Electrical, load forecasting" Butterworth Heinemann. 
[9]. Weather forecast. (2015) Port Harcourt Weather- Accuweather. [Online] Available from: http://m.accuweather.com/...weatherforecast/251990. [Accessed: 3rd March 2015].

[10]. Weather-Time and Date.(2015) Hourly Forecast for Port Harcourt, Nigeria. [Online] Available from: http://www.timeanddate.com...geria/portharcourt/hourly[Accessed: 15th September 2015].

[11]. Geographical Map.(2015) Geographical map of Port Harcourt Metropolis. [Online] Available from: http://www.google.com/geographical map/portharcourt metropolis/.. [Accessed: 3rd March 2015].

[12]. Math works. (2013) Algorithm Development. [Online] Available from:http://www.mathworks.com/solutions/algorithm development/ ?s_cid=LF_OPTA_4. [Accessed: $17^{\text {th }}$ February 2015].

[13]. Math works. (2013) Deploying and integrating load forecasting algorithm into enterprise systems. [Online] Available from:http://www.mathworks.com/product/matlab/. [Accessed: $18^{\text {th }}$ February 2015].

[14]. Math works.(2013) Neural Network Toolbox. [Online] Available from:

http://www.mathworks.com/products/neural_network/?s_cid=LF_OPTA_4[Accessed on: 19 $9^{\text {th }}$ February 2015]. 